\title{
The Anisotropy of Replicated Aluminum Foams
}

\author{
Eugeny L. Furman, ${ }^{1}$ Arcady B. Finkelstein, ${ }^{1}$ and Maxim L. Cherny ${ }^{2}$ \\ ${ }^{1}$ Department of Foundry Engineering, Ural Federal University, Ekaterinburg 620002, Russia \\ ${ }^{2}$ Department of Rare Metals, Ural Federal University, Ekaterinburg 620002, Russia \\ Correspondence should be addressed to Maxim L. Cherny; fs@kompmat.ru
}

Received 30 November 2013; Revised 23 March 2014; Accepted 24 March 2014; Published 4 May 2014

Academic Editor: Nikolaos Michailidis

Copyright (C) 2014 Eugeny L. Furman et al. This is an open access article distributed under the Creative Commons Attribution License, which permits unrestricted use, distribution, and reproduction in any medium, provided the original work is properly cited.

\begin{abstract}
The replication casting process gives the open-cell aluminum foams that can be used in many industrial applications as well as in filtering technology. The essential requirement for filters is the uniformity of filtering degree which is defined by the minimal pore size. However the structure of replication castings is often inhomogeneous and the minimal pore radius is decreasing in the direction of melt infiltration. The objective of this investigation is to study the dynamics of melt impregnation of the porous medium by vacuum suction to identify the possibility of reducing the anisotropy. Theoretical data illustrate the processes at the boundary between melt and gas medium. The experiments were carried out using the replication aluminum samples produced according to commercial technology. It was found that the permeability coefficient varies throughout the height of castings. A method for estimation of pressure on the line of melt movement was proposed. The resistance of $\mathrm{NaCl}$ layer and circular vents of the mold causes the inhomogeneity of castings. Finally the ways of minimizing the anisotropy were offered.
\end{abstract}

\section{Introduction}

Aluminum open-cell foams can be applied in a wide range of possible applications [1]. However their main field of application is filtration nowadays. The technology of replication casting creates the porous structure that differs radically from the pores obtained by powder metallurgy without using the space-holder material (Figure 1). The replicated aluminum foam obtained with vacuum suction has a porosity in the range of $50-65 \%$ which is higher than that for sintered porous structures (around 30-35\%) and lower than that for replication casting under high pressure (up to 90\%) [2, $3]$. Relatively high volumetric porosity enables the gravity sedimentation of solid particles. These factors lead to a longterm operation of filter without a significant permeability loss.

The essential requirement for filters is the uniformity of filtering degree which is defined by the minimal pore size $\left(2 r_{\text {min }}\right)$. This task can be solved with technology [3], when prebaked sodium chloride $(\mathrm{NaCl})$ layer is infiltrated with aluminum melt under high pressure. In this case the minimal pore size is determined by the time of $\mathrm{NaCl}$ baking. However the technology is rarely used commercially due to its high production cost. The technology with loose $\mathrm{NaCl}$ layer is applied by Composite Materials Ltd. (Ekaterinburg, Russia) [4] for production replicated aluminum foams in commercial scale. Here the granular sodium chloride is preheated in a furnace and is then filled into the mold and infiltrated with molten aluminum using vacuum suction. The technology is cost saving and allows producing a wide range of filters mainly for compressed air and gases. However the minimal pore radius (Figure 1) is often inhomogeneous and is decreasing in the direction of melt infiltration. The anisotropic part of the casting does not match the requirements of the customer and should be removed during machining. Therefore the objective of this investigation is to study the dynamics of melt impregnation of the porous medium by vacuum suction to identify the possibility of reducing the anisotropy.

\section{Theory}

The minimum pore radius of replicated aluminum foam is derived by the following equation [4]:

$$
r_{\min }=\frac{-3 \sigma+\sqrt{9 \sigma^{2}-8 \sigma R P \cos \theta}}{2 P},
$$



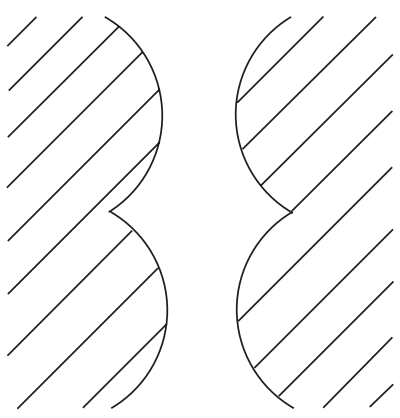

(a)

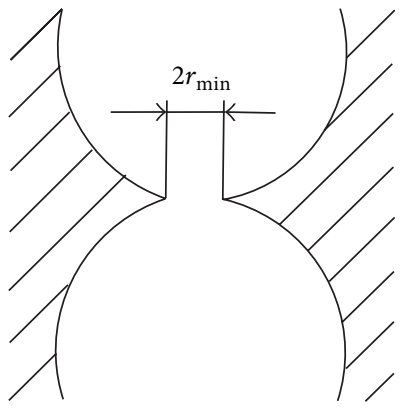

(b)

FIGURE 1: The shape of pores obtained by (a) metal sintering and (b) replication casting hatching area depicts metal.

where $\sigma$ is the surface tension of molten metal, $2 R$ is the average size of $\mathrm{NaCl}$ particles, $\theta$ is wetting angle, and $P=$ $P_{\mathrm{at}}-P_{\mathrm{ac}}+\rho g h$, where $P_{\mathrm{at}}$ is the atmospheric pressure, $P_{\mathrm{ac}}$ is the pressure of the air entrapped during melt filtration in the air collar, $\rho$ is the melt density, and $h$ is the height of liquid column.

Hydrostatic pressure will always cause the anisotropy of porous structure. Decreasing the cast height is not an acceptable solution since it will increase the production costs. The influence of hydrostatic pressure can be reduced by increasing the pressure drop during the aluminum melt infiltration.

For example, when the pressure drop equals $60000 \mathrm{~Pa}$ $(0,6 \mathrm{~atm})$, sodium chloride fraction is $0,32-0,63 \mathrm{~mm}$ and when the cast height equals $200 \mathrm{~mm}$ towards filtration, the estimated anisotropy of minimal pore radii caused by hydrostatic pressure (1) along the cast height will be $2.8 \%$, and that is technologically acceptable.

The air is entrapped by melt at the front of melt movement and as a result the air collar is forming. Subsequently the pressure at the air collar is equal to the pressure at the front of melt movement.

The main factor in anisotropy of porous structure is an alternating pressure at the front line of impregnation.

The technology includes vacuum suction of the mold filled with salt granules after melt loading on the surface of $\mathrm{NaCl}$ bed. Impregnation does not start at the moment of connection of the mold to a vacuum line. Since the salt granules are not wetted by melt, the spontaneous impregnation does not occur. So there is a certain time gap between melt loading to the surface of $\mathrm{NaCl}$ bed and start of impregnation, and this time gap is enough to form the oxide film at the border of melt and salt. The oxide film at the surface of melted aluminum has sufficiently high mechanical features; its burst pressure depends on the alloy content and for pure aluminum it is $18,000 \mathrm{~Pa}$ [5]. Therefore, the initial condition for impregnation is to provide the burst pressure of the oxide film at the border of melt and salt as follows:

$$
P(x=l)=18000 \mathrm{~Pa} .
$$

To determine the initial conditions in gaseous phase during the impregnation, it is necessary to solve the task of nonsteady air filtration in the porous medium. During gas filtration the influence of gravity and inertia can be neglected because of the low density of gases. The process of nonstationary gas flow in porous medium is described by Leibenson equation as follows:

$$
\frac{\partial P}{\partial \tau}=\frac{K}{2 \Delta \mu} \nabla^{2} P^{2}
$$

Equation (3) is a parabolic-type nonlinear equation. In case of unidirectional air filtration, (3) can be presented as

$$
\frac{\partial P}{\partial \tau}=\frac{K}{2 \Delta \mu} \frac{\partial^{2} P^{2}}{\partial x^{2}} .
$$

Let us evaluate the mold with porous material of uniform cross section and finite length (Figure 2). Initial conditions are provided by pressure $P_{0}$ in the volume of salt granules at the initial moment $(\tau=0)$ :

$$
P(x, \tau=0)=101250 \mathrm{~Pa} .
$$


The constant pressure equal to the pressure in receiver is established for the boundary between vacuum receiver and porous bed as follows:

$$
P(x=0, \tau)=P_{\text {rec }} .
$$

The velocity of gas filtration is equal to zero for boundary between melt and porous bed; thus,

$$
\frac{d P}{d x}(x=l, \tau)=0 .
$$

The terminal condition of the task is (2).

Therefore, estimation of nonstationary gas filtration is equivalent to mathematical task of integration of homogenous Fourier equation (4) with the mentioned-above initial and terminal conditions (5)-(7). The task is to determine flow capacity at the direction parallel to mold's walls at any moment. The dynamic viscosity of gas is accepted to be constant ( $\mu=$ const).

Equation (4) is nonlinear and cannot be solved analytically. Here, it is taken as a basis, the solution [6] representing an explicit schedule of the method of finite differences. Monodimensional field of pressures is an initial condition of the task of $\mathrm{NaCl}$ bed wetting out by melt.

In the process of wetting out, it comes further.

The subsequent air evacuation from $\mathrm{NaCl}$ bed to vacuum receiver is observed during the impregnation, as well as air displacement from porous medium being filled with melt. Consequently, a new source component which describes the air displacement arises in Leibenson equation for a boundary between a melt and air layer. The boundary layer of gas with volume $V$,

$$
V=S \cdot H \cdot \Delta,
$$

with height $H$ and surface $S$ (where $\Delta$ is porosity) is taken conditionally as alternating $H$ for estimation of melt line motion as

$$
\frac{d(H)}{d \tau}=-\frac{d l}{d \tau} .
$$

However, the layer's height $H$ is taken as constant $(H$ coordinate increment in porous bed) for estimation of gas filtration into outside environment. Taking account of decrease of amount of filling with coming melt is performed by annulation of boundary layer after its total fulfillment. The estimation schedule is presented (Figure 2).

Pressure in this layer is accepted to be equal through all its volume. For this layer, the Mendeleev-Clapeyron equation differentiated with respect to time can be presented as

$$
\frac{d}{d \tau}(P V)=\frac{d}{d \tau}\left(\frac{m}{M} R T\right)
$$

The process is supposed to go under isothermal conditions. Getting (10) together with (8), (9) can be transformed into the following:

$$
\left(\frac{d P}{d \tau}\right)_{l}=\frac{R T}{H \Delta S M} \frac{d m}{d \tau}+\frac{P d l}{H d \tau} .
$$

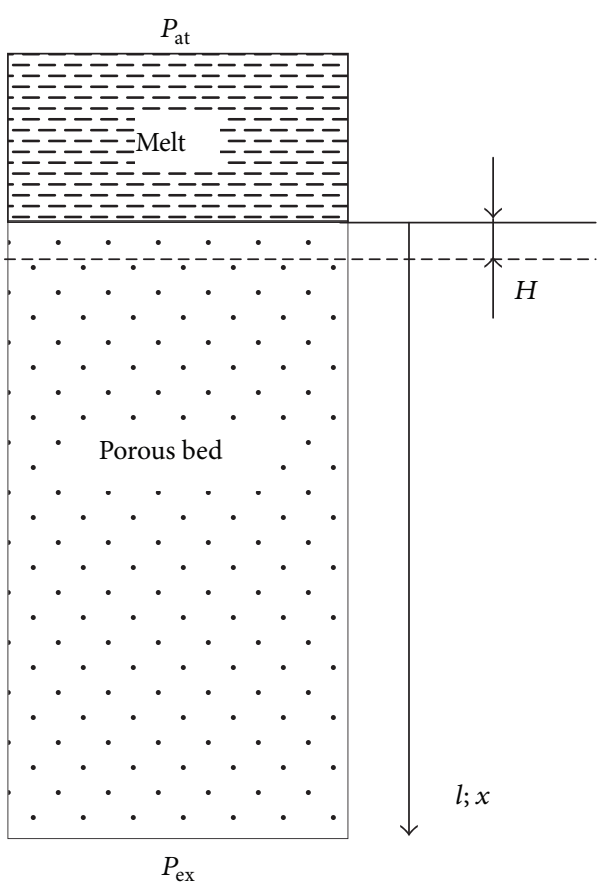

Figure 2: Principal scheme of $\mathrm{NaCl}$ porous bed impregnation.

If the boundary "melt-gas medium" was immovable $(d l / d t=0)$, (11) could be written in the form (4), but since there is a gas displacement from porous medium being filled by melt, (4) for boundary air layer on the line of melt motion is added by the source component from (11):

$$
\left(\frac{d P}{d \tau}\right)_{l}=\frac{K}{2 \Delta \mu} \frac{d^{2} P^{2}}{d x^{2}}+\frac{P d l}{H d \tau} .
$$

Equation (12) physically means that pressure in the boundary layer increases because of air displacement by melt and this pressure increases proportionally to the melt motion.

Estimation of pressure change on the line of impregnation is performed by a system of Leibenson equations (4) for gas flow with boundary conditions (6) and (12), as well as by Darcy's equation for fluid flow:

$$
\frac{d l}{d \tau}=\frac{K \cdot\left(P_{\mathrm{ex}}-P_{l}\right)}{\mu_{l} \cdot l},
$$

where $d l / d \tau$ is the average linear flow rate through porous medium, $P_{\mathrm{ex}}-P_{l}$ is the change of fluid pressure through porous medium, $\mu_{l}$ is the dynamic viscosity of the fluid, $l$ is the thickness of infiltrated porous medium along the direction of fluid flow, and $K$ is permeability of porous medium.

The boundary conditions for fluid are as follows: $P_{l}$ is determined by (12) and $P_{\mathrm{ex}}=101250 \mathrm{~Pa}$. The common initial condition for fluid and gas is $l=0$.

This solution includes reduction of grid points, while melt is filling the salt layer. Because of approximation errors, it makes no sense to keep the amount of grid points steady, that is, to perform recalculation of pressures over the coordinate, coordinate increment, and time increment. The program is written in Delphi with Borland environment. 


\section{Experimental}

In the process of impregnation, the air is entrapped by melt into the air collars. Their size is a function of residual air pressure [4]. Thus, there is a possibility to study the pressure experimentally on the line of impregnation using the dimensions of these air collars. To eliminate the influence of random collar selection, it is reasonable to use such an integrated characteristic property of porous metal as permeability coefficient. The method of the permeability coefficient evaluation is published [4].

Commercial metal mold with cylindrical cross section (Figure 3) was used for experimental samples production. The mould is placed on a vacuum receiver and is connected with it through gas valve. In commercial mold the bottom is covered by a plug, and vacuum suction is performed by air filtration through a circular vent between the mold and the plug. The gap creates sufficient hydraulic resistance. So the framed net was used instead of the full-metal plug during the experiments. The layer of cold salt was placed over the net to eliminate the filtration of melt into the vacuum receiver. Aluminum (Al) 99.95 (A95 grade in Russia) was used as melt in all experiments for proper control of physical and chemical characteristics of the process. The $\mathrm{NaCl}$ used in experiments was a commercial evaporated sodium chloride of such producers as Tyret salt mine OJSC, Uralkali. The sodium chloride was previously dried and sieved to obtain the desired granules fraction.

Inner diameter of working space of the mould is $30 \mathrm{~mm}$ and length is $140 \mathrm{~mm}$ including the metal head on the top $-40 \mathrm{~mm}$ and cold salt layer on the bottom $-20 \mathrm{~mm}$. The obtained composite cast with $80 \mathrm{~mm}$ length was cut into 8 samples with $7 \mathrm{~mm}$ thickness. The granules of $\mathrm{NaCl}$ were removed from samples by dissolving in water. Porosity of the samples was estimated by weighting, supposing that all pores in the samples are open and interconnected.

The impregnation was carried out under isothermal conditions in order to prevent changes in the physical characteristics of the melt and $\mathrm{NaCl}$ during the impregnation. The temperature of aluminum melt, $\mathrm{NaCl}$ granules, and mold was equal to $700^{\circ} \mathrm{C}$; the level of vacuum is indicated in Figures 4 and 5 .

The results of permeability coefficient's calculation compared with experimental data are presented in Figures 4 and 5 .

\section{Discussion}

The experimental results show that the permeability coefficient varies throughout the height of castings. A good repeatability of calculated and experimental results was observed with use of salt granules with fraction of $0.315-$ $0.63 \mathrm{~mm}$ (Figure 4). Slightly higher variations between estimated and experimental data are observed for $\mathrm{NaCl}$ fraction of 1.5-2.5 mm (Figure 5). But the shape of curves and depth of inhomogeneous area (about 20\% of the cast length) are identical both in theory and in practice. That proves the efficiency of proposed method for estimation of pressure on the line of melt movement.

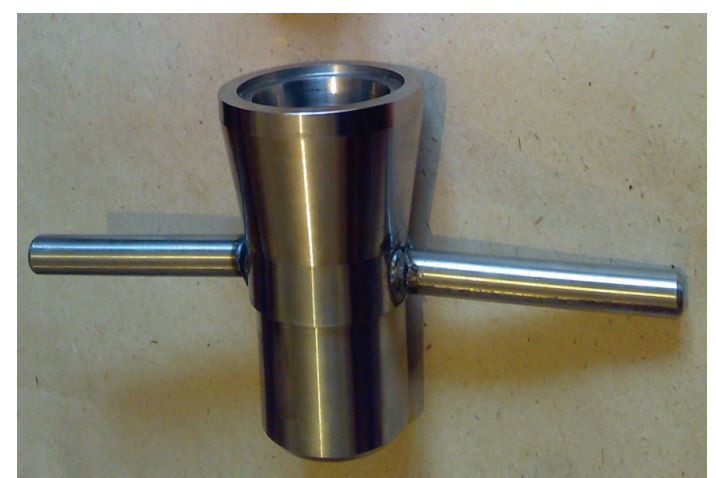

Figure 3: Commercial metal mould.

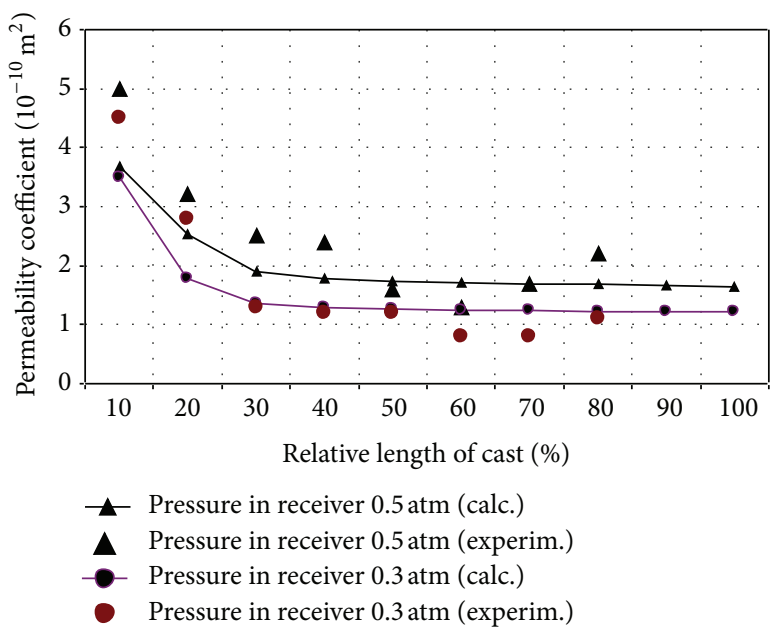

FIGURE 4: Variation of the permeability coefficient throughout the height of a cast with $\mathrm{NaCl}$ fraction of $0.315-0.63 \mathrm{~mm}$.

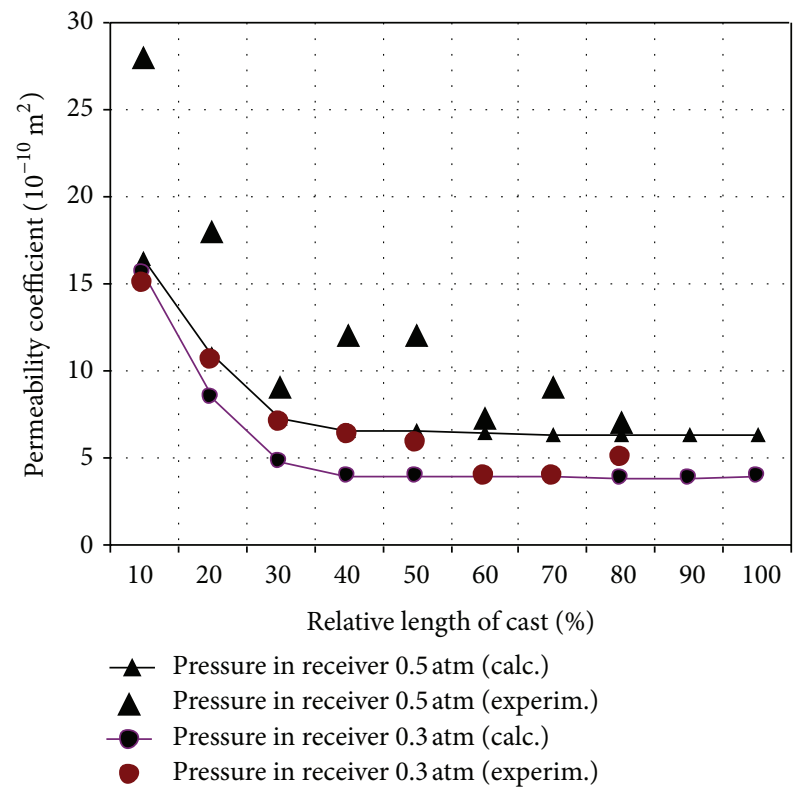

FIgURE 5: Variation of the permeability coefficient throughout the height of a cast with $\mathrm{NaCl}$ fraction of $1.5-2.5 \mathrm{~mm}$. 
The impregnation can be divided into two stages. The first stage is when the air pressure on the line of melt movement varies from oxide film burst pressure to pressure in receiver. The second stage is when the pressure on the line of melt movement is stable at the level of receiver pressure. The estimation of the size of anisotropic area under different conditions was performed to assess the capability for anisotropy control.

These estimations showed that a relative length of anisotropic area depends only on physical and chemical characteristics of gas and liquid phases (Figure 6).

The calculation was made for aluminum and zinc melts to demonstrate the effect of melt viscosity on the size of inhomogeneous area. Since zinc melt's viscosity is significantly lower than that for aluminum melt, the size of inhomogeneous area increases.

The size of inhomogeneous area does not depend on the permeability coefficient $K$. This may be explained by direct dependence on the filtration velocity from $K$ for both liquid and gas phases.

The Hagen-Poiseuille equation is applied to liquid and gas phases contemporarily to solve the task of simultaneous flow of liquid and gas in cylindrical capillary [7]. The paper [7] shows that a velocity of liquid at the initial stage depends on the length of capillary and the viscosity of liquid and gas; that is, it is determined by formation of "air pillow" on the line of liquid flow. Presented in this paper, the estimations and experiments not only prove this hypothesis [7], but also allow evaluating the absolute amount of gas pressure on the line of melt movement and taking into account the distinctive features of melt, particularly the presence of solid oxide layers and specific features of impregnation technology (applying of vacuum suction or external pressure).

\section{Conclusion}

The present paper shows that it is impossible to decrease a relative amount of inhomogeneous area theoretically. In industrial scale, as it was mentioned above, the bottom of the mould is covered not by framed net but by a fullmetal plug, and this significantly increases the total resistance of the system "NaCl layer + circular vent" (circular vent can be approximated as a part of filling). That is why the inhomogeneity is observed throughout the cast length at the direction of melt filtration in industrial.

We have found it reasonable to add a layer of fine fraction of $\mathrm{NaCl}$ granules above a basic filling layer before the impregnation [8] to stabilize the pressure. The size of $\mathrm{NaCl}$ particles in this additional layer should be as small as possible to provide higher resistance to the melt flow, but it must not be less than the capillary gaps in the basic filler to avoid the washing out by melt. Melt is casted down on the surface of fine fraction salt. During the vacuum suction the impregnation starts when a pressure on the boundary "fine fraction saltmelt" reaches the value of capillary pressure, that is, when it exceeds sufficiently a capillary pressure for the basic $\mathrm{NaCl}$ layer (or the pressure of destruction of oxide films). When the melt flows with higher resistance in fine fraction salt the

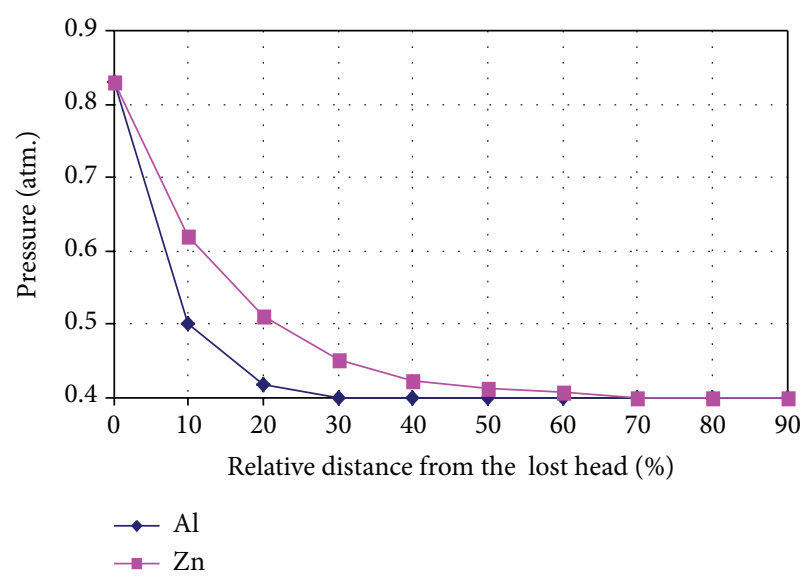

FIGURE 6: Gas pressure on the line of melt motion.

pressure in the basic salt layer becomes steady at the level of vacuum receiver pressure. Then the part of casting filled with fine fraction salt is disposed during machining. A serious restriction of this method is the impossibility to obtain the products with combination of porous and solid-metal parts.

The other method can be applied to avoid the cast inhomogeneity [9]. Here the salt layer is impregnated with melt at irreducibly small vacuum (0.2 atm). Then the form is covered by a cap and the compressed air is delivered under the cap. The obtained gradient of pressure allows adjusting the radius of air collar, as well as all performance characteristics of product within a rather wide range. This method allows producing the items that combined the porous and solidmetal parts, yet the instrumentation is more complicated in this case.

\section{Conflict of Interests}

The authors declare that there is no conflict of interests regarding the publication of this paper.

\section{References}

[1] J. F. Despois, Replicated aluminium foam, processing and properties [Doctoral Thesis], Institute of Materials, Ecole Politechnique Federale de Lausanne, 2005.

[2] J.-F. Despois and A. Mortensen, "Permeability of open-pore microcellular materials," Acta Materialia, vol. 53, no. 5, pp. 13811388, 2005.

[3] A. Jinnapat and A. Kennedy, "The manufacture and characterisation of aluminium foams made by investment casting using dissolvable spherical sodium chloride bead preforms," Metals, vol. 1, no. 1, pp. 49-64, 2011.

[4] E. Furman, A. Finkelstein, and M. Cherny, "Permeability of aluminium foams produced by replication casting," Metals, vol. 3, no. 1, pp. 49-57, 2013.

[5] E. Furman, A. Finkelstein, and Y. Yun, “Tensile strength analysis of oxide film on the aluminium melt surface.," in Proceedings of the 11th Russian Conference on Structure and properties of metal and slag melts, vol. 2, pp. 213-215, South Urals State University, Ekaterinburg, Russia, 2004. 
[6] J. S. Aronofsky and R. Jenkins, "Unsteady flow of gases through porous media," in Proceedings of the 1st US National Congress of Applied Mechanics, pp. 763-771, Illinois Institute of Technology, Chicago, Ill, USA, 1952.

[7] J. R. Ligenza and R. B. Bernstein, "The rate of rise of liquids in fine vertical capillaries," Journal of The American Chemical Society, vol. 73, no. 10, pp. 4636-4640, 1951.

[8] E. Furman, A. Finkelstein, and I. Furman, "Method of replicated foam production," Patent RU 2256530 B 22 D 25/00, 2005.

[9] B. Pastukhov, M. Mitrofanov, E. Furman et al., "Method of replicated foam production," Patent RU 1814247 B 22 D 25/00, B 22 D 27/00, C 21 C01/0, 1995. 

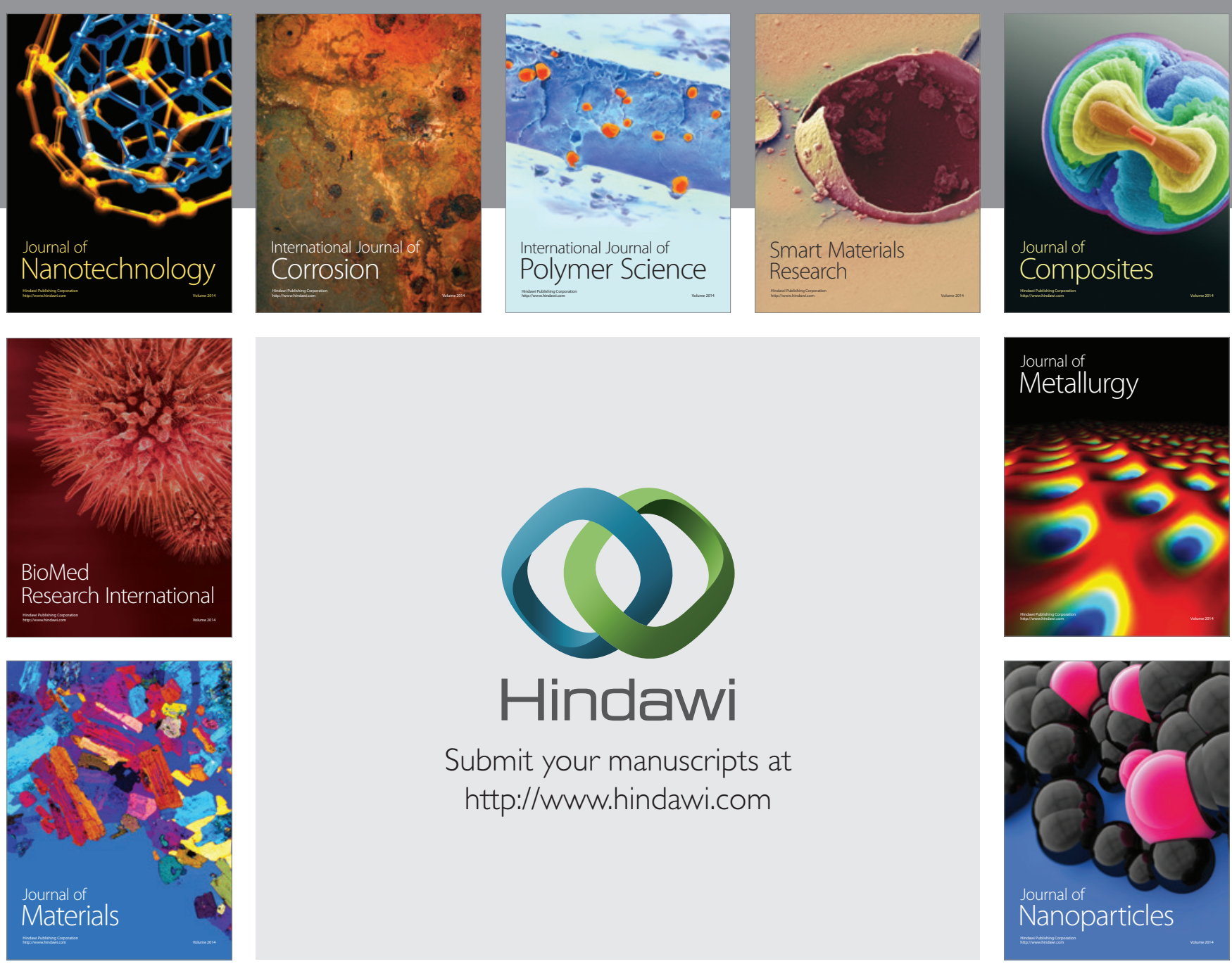

Submit your manuscripts at http://www.hindawi.com
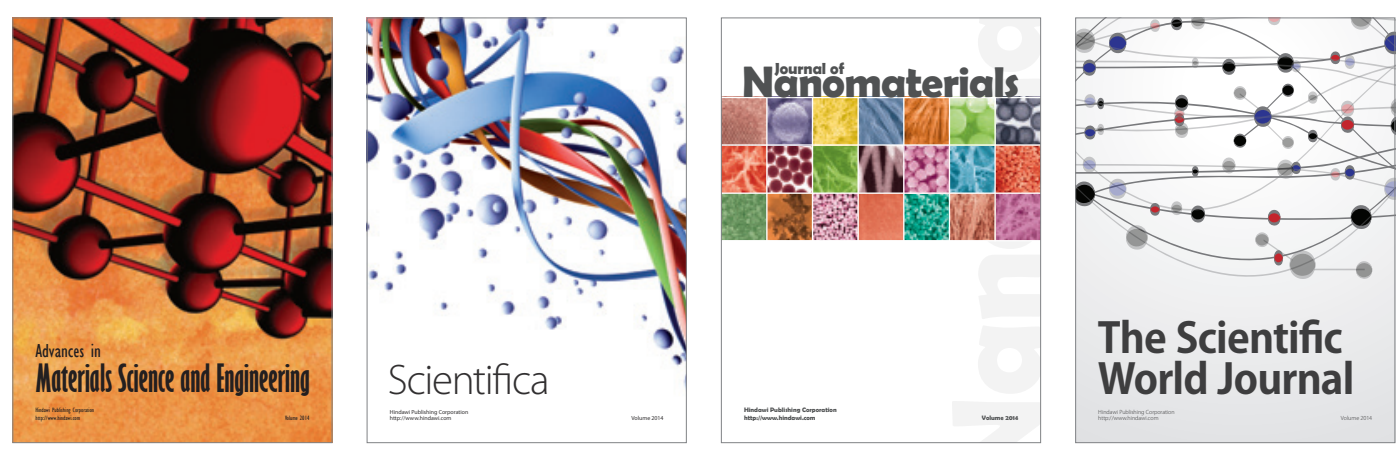

\section{The Scientific World Journal}
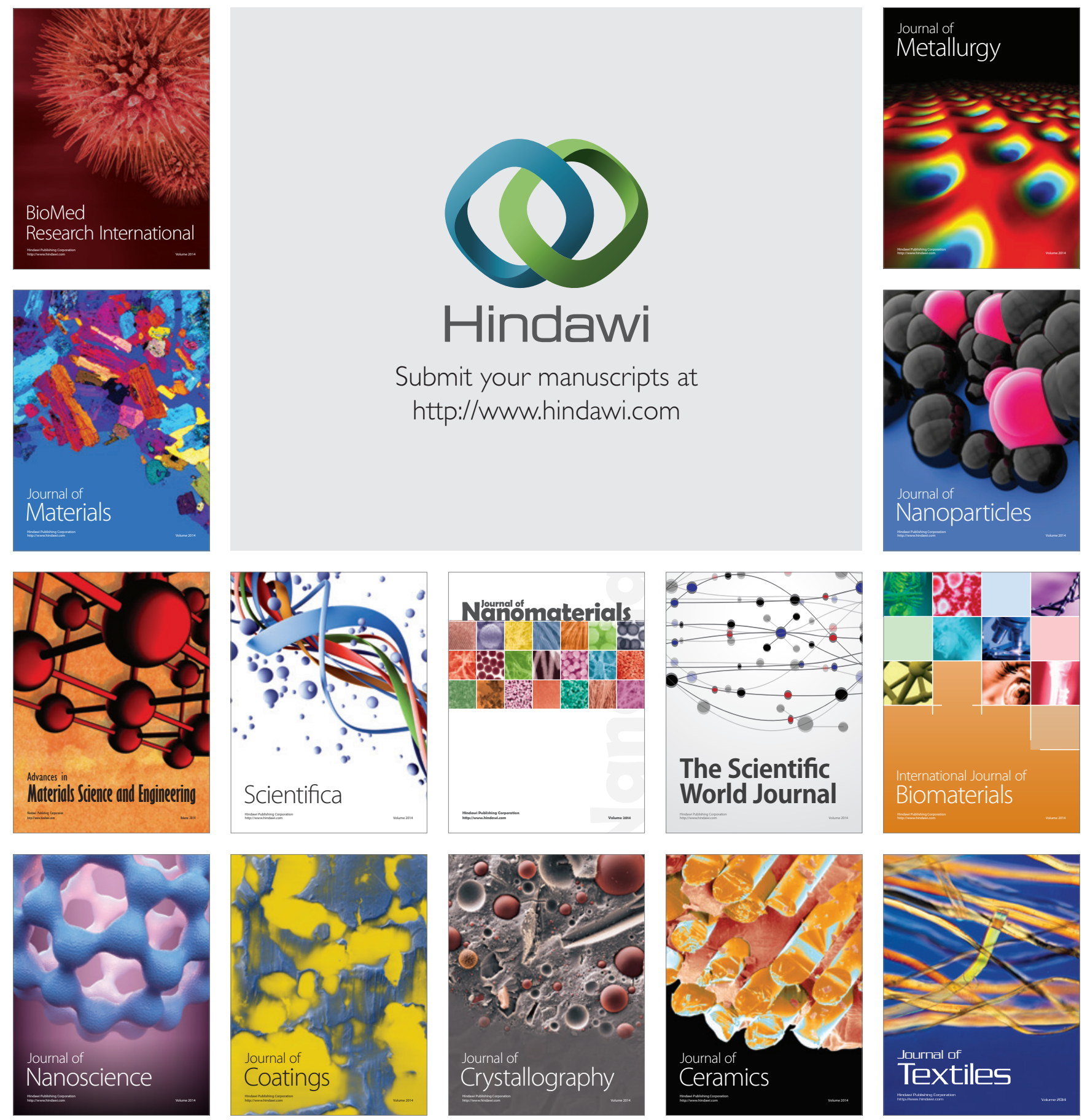